\title{
Multi-sources data fusion framework for remote triage prioritization in telehealth
}

\begin{abstract}
The healthcare industry is streamlining processes to offer more timely and effective services to all patients. Computerized software algorithm and smart devices can streamline the relation between users and doctors by providing more services inside the healthcare telemonitoring systems. This paper proposes a multi-sources framework to support advanced healthcare applications. The proposed framework named Multi Sources Healthcare Architecture (MSHA) considers multi-sources: sensors (ECG, SpO2 and Blood Pressure) and text-based inputs from wireless and pervasive devices of Wireless Body Area Network. The proposed framework is used to improve the healthcare scalability efficiency by enhancing the remote triaging and remote prioritization processes for the patients. The proposed framework is also used to provide intelligent services over telemonitoring healthcare services systems by using data fusion method and prioritization technique. As telemonitoring system consists of three tiers (Sensors/ sources, Base station and Server), the simulation of the MSHA algorithm in the base station is demonstrated in this paper. The achievement of a high level of accuracy in the prioritization and triaging patients remotely, is set to be our main goal. Meanwhile, the role of multi sources data fusion in the telemonitoring healthcare services systems has been demonstrated. In addition to that, we discuss how the proposed framework can be applied in a healthcare telemonitoring scenario. Simulation results, for different symptoms relate to different emergency levels of heart chronic diseases, demonstrate the superiority of our algorithm compared with conventional algorithms in terms of classify and prioritize the patients remotely.
\end{abstract}

Keyword: ECG; Priority; Healthcare services; Triage; Dempster-Shefer theory; Remote patient monitoring 